Peer $J$

Physical Chemistry
Submitted 26 June 2019

Accepted 31 October 2019

Published 2 December 2019

Corresponding authors

Nohad Gresh, gresh@lct.jussieu.fr Zeina Hobaika,

Zeina.hobaika@usj.edu.lb

Academic editor

Jan Jensen

Additional Information and Declarations can be found on page 14

DOI 10.7717/peerj-pchem.6

(c) Copyright

2019 El Khoury et al.

Distributed under

Creative Commons CC-BY 4.0

\section{Spectrometric and computational studies of the binding of HIV-1 integrase inhibitors to viral DNA extremities}

\author{
Léa El Khoury ${ }^{1,2,3}$, Krystel El Hage ${ }^{4,5}$, Jean-Philip Piquemal ${ }^{2,6,7}$, \\ Serge Fermandjian ${ }^{4}$, Richard G. Maroun ${ }^{2}$, Nohad Gresh ${ }^{1}$ and \\ Zeina Hobaika ${ }^{2}$ \\ ${ }^{1}$ Laboratoire de Chimie Théorique, UMR7616 CNRS, Sorbonne Université, Paris, France \\ ${ }^{2}$ UR EGP, Centre d'Analyses et de Recherche, Faculté des Sciences, Université Saint-Joseph de \\ Beyrouth, Beirut, Lebanon \\ ${ }^{3}$ Present address: Department of Pharmaceutical Sciences, University of California, Irvine, \\ Irvine, CA, USA \\ ${ }^{4}$ Chemistry and Biology Nucleo(s)tides and Immunology for Therapy (CBNIT), UMR 8601 \\ CNRS, UFR Biomedicale, Paris, France \\ ${ }^{5}$ Present address: Laboratoire Structure-Activité des Biomolécules Normales et Pathologiques, \\ INSERM U829, Université Evry-Val d'Essonne, Evry, France \\ ${ }^{6}$ Department of Biomedical Engineering, The University of Texas at Austin, Austin, TX, USA \\ ${ }^{7}$ Institut Universitaire de France, Paris, France
}

\section{ABSTRACT}

Three integrase strand transfer inhibitors are in intensive clinical use, raltegravir (RAL), elvitegravir (EVG) and dolutegravir (DTG). The onset of integrase resistance mutations limits their therapeutic efficiency. As put forth earlier, the drug affinity for the intasome could be improved by targeting preferentially the retroviral nucleobases, which are little, if at all, mutation-prone. We report experimental results of anisotropy fluorescence titrations of viral DNA by these three drugs. These show the DTG $>$ EVG $>$ RAL ranking of their inhibitory activities of the intasome to correspond to that of their free energies of binding, $\Delta G s$, to retroviral DNA, and that such a ranking is only governed by the binding enthalpies, $\Delta H$, the entropy undergoing marginal variations. We sought whether this ranking might be reproduced through quantum chemistry (QC) Density Functional Theory calculations of intermolecular interaction energies between simplified models consisting of sole halobenzene ring and the highly conserved retroviral nucleobases G4 and C16. These calculations showed that binding of EVG has a small preference over DTG, while RAL ranked third. This indicates that additional interactions of the diketoacid parts of the drugs with DNA could be necessary to further enable preferential binding of DTG. The corresponding $\Delta E_{\text {tot }}$ values computed with a polarizable molecular mechanics/dynamics procedure, Sum of Interactions Between Fragments Ab initio computed (SIBFA), showed good correlations with this $\triangle E(\mathrm{QC})$ ranking. These validations are an important step toward the use of polarizable molecular dynamics simulations on DTG or EVG derivatives in their complexes with the complete intasome, an application now motivated and enabled by the advent of currently developed and improved massively parallel software. 
Subjects Theoretical and Computational Chemistry, Biophysical Chemistry, Physical Chemistry (other)

Keywords HIV-1 Integrase, Halobenzene inhibitors, Viral DNA, Spectrometric studies, Computational studies

\section{INTRODUCTION}

HIV-1 Integrase is a key element in viral replication. In addition to its essential role in viral DNA (vDNA) integration into host genomic DNA, it is involved, directly or indirectly, in reverse transcription (Li et al., 2011), nuclear import (Engelman et al., 1995; Ikeda et al., 2004) and HIV-1 particle maturation (Balakrishnan et al., 2013; Fontana et al., 2015). As in addition integrase has no counterpart in human cells, it could represent a privileged target for the design of potent antiretroviral drugs.

The integration step is carried by a multimer of integrase proteins assembled on vDNA ends, referred to as the intasome. In a first step, denoted as $3^{\prime}$-processing, a 3'GT dinucleotide is removed from each end of the long terminal repeats (LTRs) of vDNA. This occurs in the cytoplasm within a multi-component pre-integration complex which gathers the vDNA and several viral and cellular proteins. DNA strand transfer occurs in a second step, after such a complex is chaperoned into the nucleus and results in integration of vDNA as a provirus into the host genome. This requires cutting of two phosphodiester bonds five base pairs apart on opposite strands of the host DNA and is done by free $3^{\prime}-\mathrm{OH}$ groups that were liberated following LTR processing (Bushman \& Craigie, 1991; Pommier, Johnson \& Marchand, 2005; Lesbats, Engelman \& Cherepanov, 2016). This mechanism was illustrated in two review papers, in Fig. 2 by Pommier, Johnson \& Marchand (2005) and Fig. 1 by Lesbats, Engelman \& Cherepanov (2016).

Integrase strand transfer inhibitors (INSTIs) proved to be much more effective than processing inhibitors and enabled the development of a successful class of antiretroviral drugs (Zhao et al., 2017). Three inhibitors inspired by the original diketo acids have been successively approved and are commonly used in HIV-1 treatment, namely raltegravir (RAL; MK-0518), elvitegravir (EVG; GS-9137) (Zhao et al., 2017; Grinsztejn et al., 2007; Koelsch \& Cooper, 2009) and dolutegravir (DTG; S/GSK1349572) (Grinsztejn et al., 2007; Koelsch \& Cooper, 2009; Métifiot, Marchand \& Pommier, 2013; Ballantyne \& Perry, 2013). The latter is a second generation INSTI aimed at maintaining an effective efficacy to integrase variants resistant to RAL and EVG (Anstett et al., 2017; Wainberg \& Han, 2015). A general review of integrase inhibitors was published by Pommier, Johnson \& Marchand (2005) encompassing a list of diketoacid inhibitors prior to RAL, EVG and DTG (Fig. 4, Pommier, Johnson \& Marchand, 2005).

As was the case for protease and reverse transcriptase inhibitors (Rhee et al., 2010; Tantillo et al., 1994), integrase inhibitors can generate several resistance mutations. These were recently reported following RAL, EVG and DTG treatment (Métifiot et al., 2010; Marinello et al., 2008; Hazuda et al., 2000; Wittkop et al., 2009). They affect, not only residues in direct interactions with INSTIs but also "outer-shell" residues indirectly bound (Anstett et al., 2017; Blanco et al., 2011; Chen et al., 2013). Among these are double mutations such as Q148R/H coupled to G140S/A that produce important 
A<smiles>Cc1nnc(C(=O)NC(C)(C)c2nc(C(=O)NCc3ccccc3F)c(O)c(=O)n2C)o1</smiles>

Raltegravir (RAL)
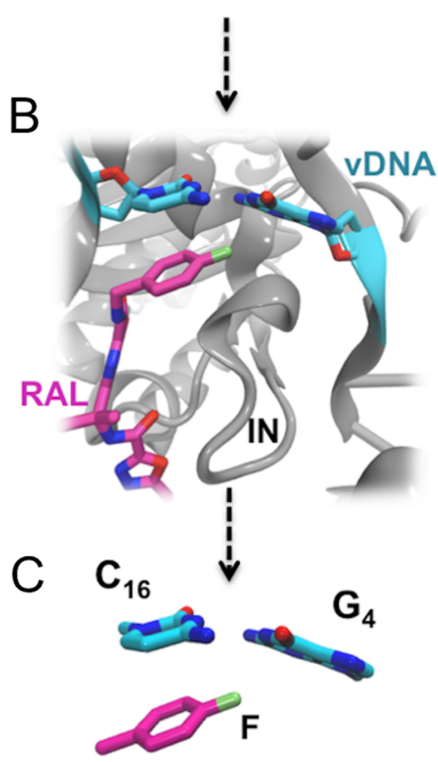

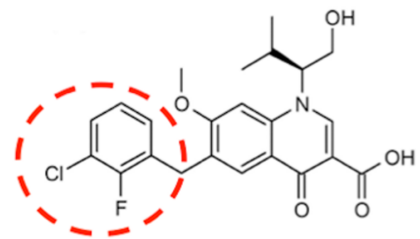

Elvitegravir (EVG)
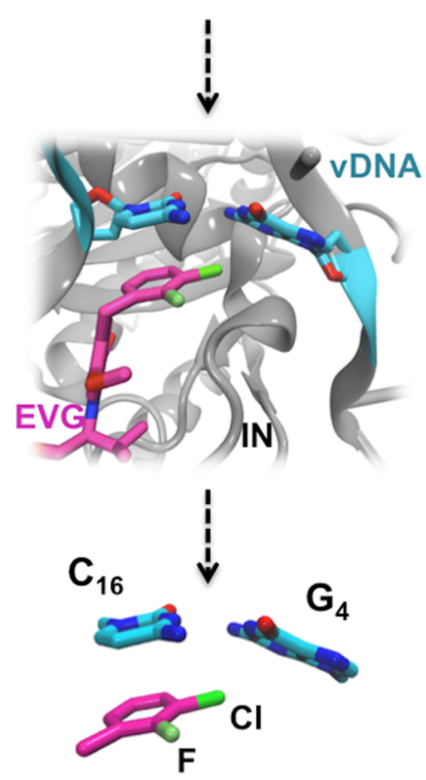

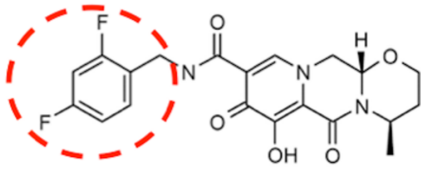

Dolutegravir (DTG)
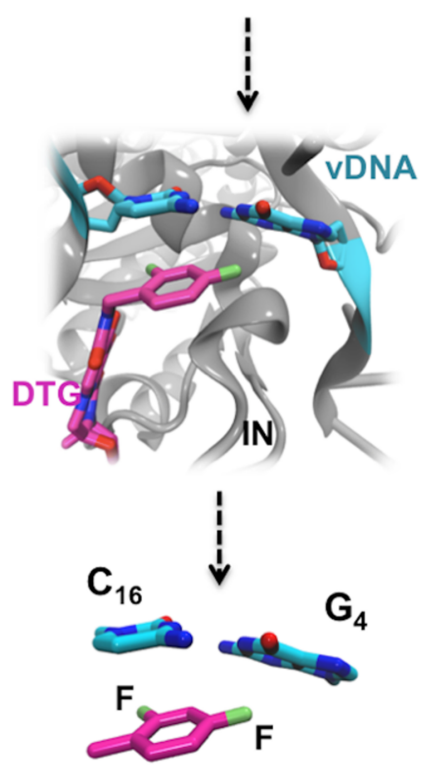

Figure 1 FDA approved Integrase strand transfer inhibitors INSTIs: raltegravir, elvitegravir and dolutegravir. (A) 2D structures of the inhibitors. The red dashed circle indicates the halobenzyl moiety. (B) High-resolution X-ray 3D structure of each inhibitor in complex with the Integrase (IN) and the viral DNA (vDNA). (C) Close-up on the interactions involving the halobenzene, cytosine 16 and guanine 4.

Full-size DOI: 10.7717/peerj-pchem.6/fig-1

synergetic effects on the efficacy of RAL, EVG and even DTG (Goethals et al., 2010;

Naeger et al., 2016).

RAL, EVG and DTG (Fig. 1) selectively bind at the interface of integrase and the vDNA ends within the intasome and have in common two distinct structural motives: (a) a large centralized pharmacophore with both keto oxygen and a coplanar neighboring oxygen coordinating the two catalytic $\mathrm{Mg}$ (II) cations, structural water molecules, and, either directly or through water, integrase residues; and (b) a halobenzyl group targeting the highly conserved 5'CpA 3'/5'TpG 3' step on the vDNA ends (Zhao et al., 2017). However, the surface and oxygen arrangement of the central pharmacophore differ among the three INSTIs, as well as the nature and position of the halogenation of their terminal aromatic ring: a single $\mathrm{F}$ is attached in para to RAL's halobenzene ring, whereas EVG has an $\mathrm{F}$ in ortho and a $\mathrm{Cl}$ in meta, and DTG has two $\mathrm{F}$ atoms in ortho and para.

Thus within the intasome, the binding of all three drugs targets both vDNA and the viral protein. In addition to the well-known established interactions with the catalytic and non-catalytic site of integrase, following the $3^{\prime}$ processing reaction, the X-ray structures of integrase-DNA-inhibitor complexes (Fig. 1B) show that the halogenated benzene ring 
stacks over the $\mathrm{C}$ base (C16) upstream, while the $\mathrm{C}-\mathrm{X}$ bond points toward the center of the G base (G4) downstream of the second strand (Hare et al., 2010a, 2010b, 2011).

On another note, it was reported that, compared with RAL and EVG, DTG displays a more potent in vitro anti-HIV activity and a distinct resistance profile (Anstett et al., 2017; Wainberg \& Han, 2015; Vandekerckhove, 2010; Osterholzer \& Goldman, 2014). Furthermore, we have ourselves reported that an increase in the drug-vDNA complex stability correlates with an increase in drug activity and a decrease in viral resistance (Anstett et al., 2017; Hare et al., 2010b, 2011), highlighting the important contribution of the vDNA end recognition for the binding affinity of INSTIs to the intasome (Hobaika et al., 2010; El Khoury et al., 2017; Ammar et al., 2012, 2016). In this connection, we have shown a narrow correlation between the strongest DTG-DNA affinity and DTG's highest barrier to resistance (El Khoury et al., 2017).

Maximizing shape complementarity at the integrase-vDNA-inhibitor surface could serve as a guiding principle for the development of new INSTIs (Zhao et al., 2017; Hare et al., 2010a; Hobaika et al., 2009, 2010). An important feature of second generation INSTIs consists into their increased contacts not only with protein active and non-active site, but also with processed vDNA, right before the strand transfer step. This is supported by the fact that the sequence of the nucleic bases at the ends of the LTR represents stringent requirements concerning retroviral integration and that there is no evidence of mutations in the LTRs that could lead to resistance to INSTIs (Hobaika et al., 2009; LaFemina, Callahan \& Cordingley, 1991; Sherman, Dickson \& Fyfe, 1992; Zargarian et al., 2003).

Could attempts to design novel INSTIs with enhanced affinities focus on the vDNA, which would render the new inhibitors much less sensitive to mutations occurring on integrase?

As a continuation of our previous studies, we experimentally measure the $\Delta G$ values for the binding of RAL, EVG and DTG to vDNA. This is done by fluorescence anisotropy experiments at three different temperatures. We also analyze, by both ab initio quantum chemistry (QC) and polarizable molecular mechanics/dynamics, the intermolecular interaction energies of their halobenzyl rings with G4 and C16. The individual energy contributions of $\Delta E(\mathrm{QC})$ are also compared to their Sum of Interactions Between Fragments $\mathrm{Ab}$ initio computed (SIBFA) counterparts. Such analyses and validations of inhibitor interactions within the core of vDNA binding site constitute a necessary step toward long-duration polarizable molecular dynamics of drug-intasome complexes.

An outstanding feature of the CX ring in halobenzenes, discovered on the basis of QC (Clark et al., 2007; Politzer, Murray \& Clark, 2010) is the existence of a zone of electron depletion along the extension of the bond with a magnitude increasing along the series $\mathrm{F}>\mathrm{Cl}>\mathrm{Br}>\mathrm{I}$. This "sigma-hole" goes along with a zone of electronic build-up on a cone around the halogen. It has been earlier shown that atom-centered point charges used in "classical" force-fields cannot account for the impact of the sigma-hole on the Coulomb electrostatic contribution $E_{\mathrm{C}}$ : this could be only partly remedied upon resorting to an additional fictitious atom prolonging the $\mathrm{CX}$ bond with a partial charge and a distance to the X bearer that have to be fit on the basis of QC calculations (Ibrahim, 2011; Jorgensen \& Schyman, 2012; Kolar \& Hobza, 2012). On the other hand, anisotropic potentials such as SIBFA, with distributed atomic multipoles up to quadrupoles, were shown to closely 


\section{5’ A C T G C TA G A G AT TTT C C T}

\section{T-F}

\section{3' HO-A C G A T C T C T A AAA G G T}

1514

1

Figure 2 LTR32 is a linear oligonucleotide sequence designed to adopt a double strand hairpin structure in solution upon folding around a loop created by a purposely added thymine triplet (TTT in green) with the sensitive fluorescein reporter (F, in orange) grafted to its central T. The latter allows fluorescence studies in solution at low concentrations. The stem that reproduces the $3^{\prime}$ processed LTR end comprises a 17-nucleotide strand and a 15-nucleotide strand corresponding to the unreactive strand and the reactive strand, respectively. Their pairing leaves an unpaired dinucleotide $5^{\prime} \mathrm{AC} 3^{\prime}$ at the $5^{\prime}$ end on the unreactive strand. In each strand the nucleotide numbering goes from the $5^{\prime}$ to the $3^{\prime}$ extremity. The highly conserved doublet of base pairs, here numbered C14-G4 and A15-T3, is colored in red. The base pair C14-G4 is the equivalent of C16-G4 mentioned in the text based on the PDB entries used in the computations.

Full-size DOI: 10.7717/peerj-pchem.6/fig-2

account for the impact of the sigma-hole along the $\mathrm{F}, \mathrm{Cl}$ and $\mathrm{Br}$ series on the magnitude of $E_{\mathrm{C}}$ both along and around the CX- bond without extra calibration effort (El Hage et al., 2013). Along these lines, recent work showed that another possibility, resorting to a distributed charge model to reproduce the local quadrupole around the CX could also enable to account for the impact of the sigma-hole on $E_{\mathrm{C}}$ (Devereux et al., 2014; El Hage et al., 2016). An additional incentive to validate SIBFA in the present work is the perspective of its applications to the entirety of the inhibitor-intasome complex, in which the presence of two $\mathrm{Mg}(\mathrm{II})$ cations close to one another and of structured water molecules render it preferable to resort to polarizable potentials than to "classical," non-polarizable ones.

At this stage we do not intend to compute true $\Delta G$ s to compare the binding affinities of the three inhibitors to vDNA and a fortiori to the intasome. These would only be meaningful at the outcome of long-duration Molecular Dynamics, and such an outcome could be very sensitive to the accuracy of the intermolecular potential. As a first step toward this evaluation, we focus here on the sole halobenzene-G4/C16 interactions expressed in terms of actual enthalpies of binding and evaluate: (a) how well the ranking of $\triangle E(\mathrm{QC})$ intermolecular interaction energies in the ternary complexes compares to the experimental binding enthalpy ranking; (b) how well the magnitudes of $\Delta E_{\text {tot }}$ (SIBFA) compare to those of $\triangle E(\mathrm{QC})$ in the three complexes and if they have the same ranking.

\section{MATERIALS AND METHODS}

\section{DNA sample and inhibitors}

The oligonucleotide LTR32 (Fig. 2) was purchased from Eurogentec (Belgium). It was designed to adopt a folded double-stranded hairpin structure even under the low concentrations $\left(10^{-9}\right.$ to $\left.10^{-5} \mathrm{M}\right)$ used in fluorescence anisotropy experiments. RAL, EVG and DTG were purchased from AdooQ and Medchemexpress, respectively and their structures are represented in Fig. 1A. 
LTR32 is a linear oligonucleotide sequence designed to adopt a double strand hairpin structure in solution upon folding around a loop created by a purposely added thymine triplet (TTT in green) with the sensitive fluorescein reporter (F, in orange) grafted to its central T. The latter allows fluorescence studies in solution at low concentrations. The stem that reproduces the $3^{\prime}$ processed LTR end comprises a 17 -nucleotide strand and a 15-nucleotide strand corresponding to the unreactive strand and the reactive strand, respectively. Their pairing leaves an unpaired dinucleotide $5^{\prime} \mathrm{AC} 3^{\prime}$ at the $5^{\prime}$ end on the unreactive strand. In each strand the nucleotide numbering goes from the $5^{\prime}$ to the $3^{\prime}$ extremity. The highly conserved doublet of base pairs, here numbered C14-G4 and A15-T3, is colored in red.

\section{Fluorescence measurements}

Thermodynamic parameters of ligand-processed DNA complexes were identified using fluorescence anisotropy (Heyduk \& Lee, 1990; Hill \& Royer, 1997) on a Jobin-Yvon Fluoromax II instrument. RAL was purchased from AdooQ and EVG and DTG were purchased from Medchemexpress. The LTR32 oligonucleotide, reproducing the processed vDNA, was purchased from Eurogentec (Belgium). It contains a thymine loop bearing the fluorescein reporter for fluorescence studies. During titrations, labeled DNA (LTR32) was dissolved in phosphate buffer $(10 \mathrm{mM}, \mathrm{pH} 6, \mathrm{I}=0.1)$ and placed in thermally jacketed quartz cells (one $\mathrm{cm}$ ) at 5,15 and $25{ }^{\circ} \mathrm{C}$; increasing concentrations of the inhibitors (RAL, EVG or DTG) were then added. The excitation was recorded at $488 \mathrm{~nm}$ and the emission at $516 \mathrm{~nm}$. The equilibrium dissociation constants $\left(K_{\mathrm{d}}\right)$ were determined with GraphPAD Prism 5 applying the non-linear regression (curve fit)-Least square procedure. This analysis led to the calculation of binding free energies using the following equation: $\Delta G=-R T \ln \left(1 / k_{\mathrm{d}}\right)$. In order to transform the obtained $\Delta G$ into $\Delta H$ and $\Delta S$,

we graphed a linear function: $\Delta G=\Delta H-T \Delta S$ for each DNA-ligand complex (LTR32-RAL, LTR32-EVG, and LTR32-DTG); $\Delta G$ (kcal/mole) is the binding free energy at 5,15 and $25^{\circ} \mathrm{C}$ and $T$ is the respective temperature in Kelvin. The fluorescence anisotropy profiles of RAL, EVG and DTG are displayed in Figs. S1-S3 respectively.

\section{PDB entries}

In the used computational approaches, all complexes were extracted from the X-ray structures of the PFV intasome (IN-viral DNA- $\mathrm{Mg}^{2+}$ ) in complex with EVG (Hare et al., 2010a) (PDB code: 3L2U), DTG (Hare et al., 2011) (PDB code: 3S3M) and RAL (Hare et al., 2010b) (PDB code: 3OYA).

\section{Ab-initio QC computations}

The systems were first energy-minimized at the correlated level using the dispersioncorrected B97-D3 functional by Goerigk \& Grimme (2011) and the cc-pVTZ basis set (Dunning, 1989; Feller, 1996) with the Gaussian 09 (G09) software (Frisch et al., 2009). The $\mathrm{C}_{1}$, atoms of $\mathrm{G} 4$ and $\mathrm{C} 16$ were replaced by an $\mathrm{H}$ atom and the $\mathrm{H}-\mathrm{N}_{9}$ and $\mathrm{H}-\mathrm{N}_{1}$ bonds were set at $1.0 \AA$, retaining the $\mathrm{N}$ in the position it occupies in DNA in the corresponding $\mathrm{X}$-ray structure. During minimization, these two bonds were held in place. This constraint 
was set to account for the anchoring of G4 and C16 in the DNA backbone. At the outcome of energy-minimization, we could verify that the G4-C16 base-pair intermolecular interaction energies had equal values in the three complexes. This was not the case without base-pair relaxation, the intermolecular G4-C16 interaction energy being three $\mathrm{kcal} / \mathrm{mole}$ less favorable in the EVG ternary complex than in the RAL and DTG ones. Thus, such a relaxation, even though partial, removes any bias that would disfavor the EVG complex with respect to the two other ones. Similarly, the $\mathrm{C}$ atom connecting the halobenzene ring to the diketoacid was replaced by an $\mathrm{H}$ atom, the $\mathrm{CH}$ bond being set at $1.09 \AA$, the halobenzene $\mathrm{C}$ occupying the same position as in the crystal structure. We found it necessary to also retain the $\mathrm{HC}$ bond in place, so that minimization of the halobenzene ring was limited to actual rotations around this bond along with minor intramolecular ring geometry relaxation. It was found that otherwise for the DTG complex a translation of the halobenzene ring could occur with full stacking underneath G4, but this would be prevented in the presence of the anchoring diketoacid entity which would then undergo unfavorable steric interactions with DNA. Figures $3 \mathrm{~A}-3 \mathrm{C}$ give representations of the three ternary complexes, with a superimposition of the starting and final structures.

At the outcome of energy-minimization, single-point calculations were performed with the B97D3 functional including the correction for basis set superposition error (BSSE) (Boys \& Bernardi, 1970; Simon, Duran \& Dannenberg, 1996). They were also done with the B3LYP-D3 (Goerigk \& Grimme, 2011), wB97X-D (Mardirossian \& Head-Gordon, 2014) and B97-D functionals (Grimme, 2006). Energy-decomposition analyses (EDA) were performed using the ALMOEDA procedure (Khaliullin et al., 2007; Horn, Mao \& Head-Gordon, 2016) with the QChem software (Shao et al., 2015).

\section{SIBFA computations}

The intermolecular interaction energy $\left(\Delta E_{\text {tot }}\right)$ is computed as the sum of five contributions: electrostatic multipolar $\left(E_{\mathrm{MTP}}\right)$, short-range repulsion $\left(E_{\mathrm{rep}}\right)$, polarization $\left(E_{\mathrm{pol}}\right)$, charge transfer $\left(E_{\mathrm{CT}}\right)$ and dispersion $\left(E_{\text {disp }}\right)$ (Gresh et al., 2007). $E_{\mathrm{MTP}}$ is computed with distributed multipoles derived from the QC molecular orbitals of the individual ligands (Stone, 1981; Stone \& Alderton, 1985; Vigné-Maeder \& Claverie, 1988), augmented with penetration (Piquemal, Gresh \& Giessner-Prettre, 2003). The anisotropic polarizabilities are distributed on the centroids of the localized orbitals using the Garmer and Stevens procedure (Garmer \& Stevens, 1989) coded in the GAMESS software (Schmidt et al., 1993).

$E_{\text {rep }}$ and $E_{\mathrm{CT}}$ are computed using representations of the molecular orbitals on the chemical bonds and the lone-pairs. $E_{\text {disp }}$ has an expansion into $1 / R^{6}, 1 / R^{8}$, and $1 / R^{10}$ along with an exchange-dispersion component (Creuzet, Langlet \& Gresh, 1991).

\section{RESULTS}

We have previously reported that the strong INSTIs bind tightly to the ends of the 3'-processed vDNA, at the LTR ends (El Khoury et al., 2017); thus, justifying their function as a blocker of the strand transfer step (Hare et al., 2010a). Table 1 reports the $K_{\mathrm{d}}$ values for the binding of RAL, EVG and DTG to the LTR32 oligonucleotide, as determined by fluorescence anisotropy titrations in a phosphate buffer at 5,15 and $25{ }^{\circ} \mathrm{C}$. 


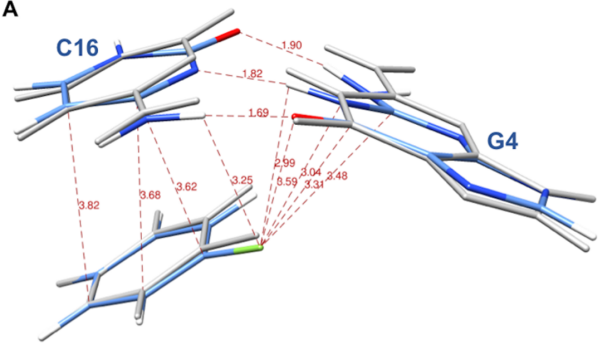

B

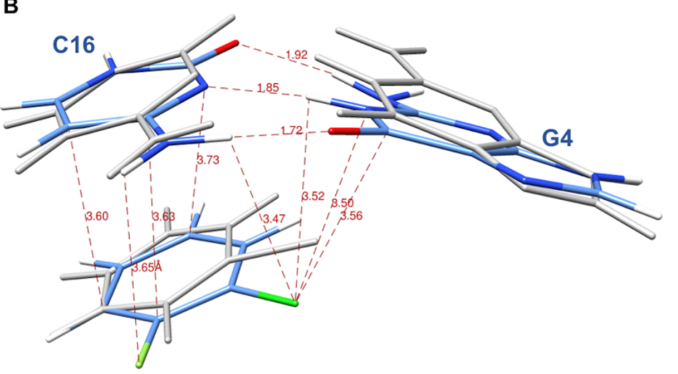

C

Figure 3 Representation of the complex of G4/C16 with the halobenzene rings of RAL (A), EVG (B) and DTG (C) at the outcome of restrained energy-minimization, with relevant intermolecular distances. The starting X-ray structures are shown in white.

Full-size DOI: 10.7717/peerj-pchem.6/fig-3

\begin{tabular}{llll}
\hline \multicolumn{2}{c}{ Table 1 Dissociation constants $\left(\boldsymbol{K}_{\mathbf{d}} \mathbf{s}\right)$ of LTR32-INSTIs interactions. } & \\
\multicolumn{1}{l}{ Temperature } & RAL & EVG & DTG \\
\hline 278 & $5.90 \mathrm{E}-09$ & $9.50 \mathrm{E}-11$ & $1.63 \mathrm{E}-12$ \\
288 & $7.69 \mathrm{E}-09$ & $1.44 \mathrm{E}-10$ & $2.74 \mathrm{E}-12$ \\
298 & $1.26 \mathrm{E}-08$ & $2.65 \mathrm{E}-10$ & $6.07 \mathrm{E}-12$ \\
\hline Note: & & \\
Temperatures in Kelvin, $K_{\mathrm{d}}$ values in molar. & &
\end{tabular}

The fluorescence anisotropy titration curves of LTR32 for increasing concentrations of drugs are reported in Figs. S1-S3. Those of LTR32 by DTG and EVG at $5{ }^{\circ} \mathrm{C}$ already reported in El Khoury et al. (2017), are added to this study for completeness. The DTG > EVG $>$ RAL $K_{\mathrm{d}}$ ranking of inhibitor-vDNA binding affinities is the same as the one reported for their binding to the complete intasome (Hightower et al., 2011).

We have further unraveled the enthalpy and entropy components of the free energies of LTR32-INSTI complexation. The $\Delta G, \Delta H$ and $\Delta S$ values are reported in Tables 2A-2C. These results show the affinity ranking of the three inhibitors for end vDNA to be dominated by the enthalpy component. This finding is fully consistent with the microcalorimetry study reported by Chaires (2006) which covered 26 DNA ligands and led to the conclusion that formation of DNA-intercalator complexes is enthalpy-driven, while that of DNA-groove binder complexes is entropy-driven. RAL-LTR32, EVG-LTR32 and DTG-LTR32 interactions are characterized by a mean $\Delta H / \Delta G$ ratio in the 1.3-1.4 range. This is within the $0.83-1.97 \Delta H / \Delta G$ ratio range, considered as a signature of an enthalpy-dominated interaction. 
Table 2 Dissociation constants and thermodynamic values of DNA-INSTI binding.

\begin{tabular}{llll} 
Temperature & $\boldsymbol{\Delta}$ & $\boldsymbol{\Delta} \boldsymbol{H}$ & $\boldsymbol{T} \boldsymbol{S}$ \\
\hline (A) Thermodynamic contributions of LT32-raltegravir interactions. & & & \\
278 & -10.07 & -14.16 & 4.09 \\
288 & -10.29 & -14.53 & 4.24 \\
298 & -10.37 & -14.75 & 4.39 \\
(B) Thermodynamic contributions of LT32-elvitegravir interactions. & & & \\
278 & -12.27 & -16.36 & 4.09 \\
288 & -12.48 & -16.71 & 4.23 \\
298 & -12.56 & -16.94 & 4.38 \\
(C) Thermodynamic contributions of LT32-dolutegravir interactions. & & & 4.07 \\
278 & -14.43 & -18.50 & 4.22 \\
288 & -14.66 & -18.88 & 4.37 \\
298 & -14.72 & -19.08 & 4.37 \\
Note: & & &
\end{tabular}

The above experimental results are an incentive for SIBFA polarizable molecular dynamics simulations of complexes of various halogenated drugs with retroviral DNAs, which should benefit from the massively parallel Tinker-HP software, co-developed in one of our Laboratories (Lagardère et al., 2018). We deemed it necessary, however, to perform a prior validation in addressing the question: to which extent would the binding of the drug halobenzyl rings to $\mathrm{G} 4$ and $\mathrm{C} 16$ be accountable for the DTG $>$ EVG $>$ RAL ranking, and how well could the outcome from high-level QC computations be accounted for by the SIBFA polarizable molecular mechanics procedure?

The considered complexes have small sizes and, for the present purposes, energy-minimizations bore on the sole halobenzyl ring. An evaluation of the SIBFA accuracy is nevertheless mandatory, as there would be little hope that inconsistencies between the SIBFA and QC results at this early stage could be obliterated or restored by subsequent large-scale molecular dynamics simulations on the entire drug-IN-vDNA complex. It also is in line with our previous analyses on the binding of a series of mono- and poly-halogenated rings to G4/C16 and the sensitivity of $\Delta E$ and its individual contributions to diverse chemical substitutions (El Hage et al., 2014, 2015).

Table $3 \mathrm{~A}$ lists the $\Delta E(\mathrm{QC})$ and $\Delta E_{\text {tot }}(\mathrm{SIBFA})$ values, and Table $3 \mathrm{~B}$ reports the ALMOEDA contributions to $\Delta E(\mathrm{~B} 97-\mathrm{D})$ and $\Delta E(\omega \mathrm{B} 97 \mathrm{X}-\mathrm{D})$ and to $\Delta E_{\text {tot }}(\mathrm{SIBFA})$. The chloro-fluorobenzene ring of EVG is found to be favored over the difluoro ring of DTG in their ternary complexes, by a small margin. It amounts to 1 and $1.6 \mathrm{kcal} / \mathrm{mole}$ out of 40 with the B97-D3 BSSE-uncorrected and corrected functional, respectively, and is reduced to 0.4 and $1.0 \mathrm{kcal} / \mathrm{mol}$ with the B3LYP-D3 BSSE-uncorrected and corrected functionals. The ALMOEDA differences are smaller, namely 0.1 (B97D) and $0.7 \mathrm{kcal} / \mathrm{mole}$ ( $\omega$ B97X-D). Such differences could simply reflect an inherent preference for the G4/C16 base pair of the chloro-fluorobenzene ring rather than the difluorobenzene ring. The DTG ring is on the other hand, consistently favored over the RAL one. The differences are 
Table 3 Values $(\mathrm{kcal} / \mathrm{mol}$ ) of the intermolecular interaction energies in the $\mathrm{G} 4 / \mathrm{C} 16 /$ halobenzene complexes.

(A)

\begin{tabular}{llllll}
\hline Procedure & B97D3 & B3LYPD3 & wB97D & B97D & SIBFA \\
\hline RAL complex & & & & & \\
$\Delta E_{\text {tot }}$ & $-41.4^{\mathrm{a}}$ & $-42.7^{\mathrm{a}}$ & $-38.5^{\mathrm{c}}$ & $-36.2^{\mathrm{c}}$ & -38.6 \\
& $-37.2^{\mathrm{b}}$ & $-38.7^{\mathrm{b}}$ & & & \\
EVG complex & & & & & -42.0 \\
$\Delta E_{\text {tot }}$ & $-43.3^{\mathrm{a}}$ & $-44.1^{\mathrm{a}}$ & $-39.9^{\mathrm{c}}$ & $-37.2^{\mathrm{c}}$ & \\
& $-39.6^{\mathrm{b}}$ & $-40.5^{\mathrm{b}}$ & & & \\
DTG complex & & & & & -39.3 \\
$\Delta E_{\text {tot }}$ & $-42.3^{\mathrm{a}}$ & $-43.7^{\mathrm{a}}$ & $-39.2^{\mathrm{c}}$ & & \\
& $-38.0^{\mathrm{b}}$ & $-39.5^{\mathrm{b}}$ & & & \\
\hline
\end{tabular}

(B)

B97D

$\omega B 97 X-D$

HF

SIBFA

\section{RAL complex}

$E_{\text {MTP }}$

$-47.5$

$E_{\text {rep }}$

$E_{1}$

$E_{\text {pol }}$

$-6.1$

$-6.1$

$-13.0$

$-13.1$

50.9

$E_{\mathrm{ct}}$

$-17.1$

$-14.1$

3.5

$\Delta E$

$E_{\text {disp }}$

$\Delta E_{\text {tot }}$

$-36.1$

$-38.5$

$-11.5$

EVG complex

$E_{\mathrm{MTP}}$

$E_{\text {rep }}$

$E_{1}$

$E_{\text {pol }}$

$-8.7$

$-12.3$

$-14.1$

$-12.5$

$-9.3$

$-6.8$

$E_{\mathrm{ct}}$

$-16.2$

$-13.3$

$\Delta E$

$E_{\text {disp }}$

$\Delta E_{\text {tot }}$

$-37.1$

$-39.9$

$-14.8$

$-23.8$

$-38.5$

DTG complex

$E_{\mathrm{MTP}}$

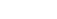

$$
-19.7
$$$$
-38.5
$$

$E_{\text {rep }}$

$E_{1}$

$-6.3$

$-11.3$

$-46.3$

$E_{\text {pol }}$

$-13.2$

$-13.3$

$-17.5$

$-14.6$

3.9

46.4

$E_{\mathrm{ct}}$

$\Delta E$

$E_{\text {disp }}$

\section{$-13.0$}

0.1

$-11.2$

$-8.6$

$-6.5$

$-17.8$

$-17.5$

$-24.5$

$-42.1$

$\Delta E_{\text {tot }}$

Note:

aSSE-uncorrected.

BSSE-corrected.

Values from ALMOEDA ED. 
more stable, amounting to $0.8-1$ and $\mathrm{kcal} / \mathrm{mole}$ with all four functionals. They may simply reflect the gain in energy brought about by the second fluorine substituent.

We have computed the zero-point vibration energies (ZPE) in the three minimized complexes. For each ligand, the differences of ZPE between the ternary complex and the three separate monomers were the same, $2.2 \mathrm{kcal} / \mathrm{mole}$. ZPE is thus not expected to impact the $\Delta E(\mathrm{QC})$ ranking.

For consistency, in each complex, the present SIBFA calculations were done with the same internal geometries of the three monomers as the B97-D3 minimized ones. $\Delta E_{\text {tot }}(\mathrm{SIBFA})$ reproduces the $\mathrm{QC}$ ranking. It has values consistently intermediate between the BSSE-uncorrected and corrected $\triangle E$ (B97-D3) ones, and this is also the case with respect to $\triangle E(\mathrm{~B} 3 \mathrm{LYP}-\mathrm{D} 3)$ values concerning the difluoro $\mathrm{DTG}$ and monofluoro rings of RAL. $\Delta E_{\text {tot }}($ SIBFA) appears to overestimate the EVG vs DTG ring preference, namely $2.5 \mathrm{kcal} / \mathrm{mole}$, while such a difference does not exceed $1.6 \mathrm{kcal} / \mathrm{mole}$ at the DFT level.

Table $3 \mathrm{~B}$ lists the values of the ALMOEDA energy contributions to $\Delta E(\mathrm{~B} 97 \mathrm{D})$ and $\Delta E$ ( $\omega$ B97X-D) along with the SIBFA ones. $\triangle E(\omega \mathrm{B} 97 \mathrm{X}-\mathrm{D})$ has $2.1-2.8 \mathrm{kcal} / \mathrm{mole}$ larger magnitudes than $\Delta E(\mathrm{~B} 97 \mathrm{D})$. While both functionals have in each complex very similar values of $E_{\mathrm{pol}}$, they differ significantly regarding $E_{1}$ and $E_{\mathrm{ct}} . E_{1}$ is app. Five kcal/mole larger with the $\omega \mathrm{B} 97 \mathrm{X}-\mathrm{D}$ than the B97-D functional, while $E_{\mathrm{ct}}$ is smaller by three kcal/mole. $\Delta E_{\text {tot }}(\mathrm{SIBFA})$ is very close to $\Delta E(\omega \mathrm{B} 97 \mathrm{X}-\mathrm{D})$ for the two fluorinated derivatives but larger by $2.2 \mathrm{kcal} / \mathrm{mole}$ for the fluoro-chlorobenzene ring of EVG. The comparison should not be carried out further at this stage, since (a) the present SIBFA calculations resort to HF-derived multipoles and polarizabilities, $E_{\text {disp }}$ being a post-HF correction; (b) all DFT-ALMOEDA contributions embody a van der Waals kernel encompassing correlation/dispersion effects, and there is not explicit dispersion contribution.

Consideration of the HF results for both mono- and difluoro rings of RAL and DTG shows unusually large differences between the magnitudes of $\triangle E(\mathrm{HF})$ and $\triangle E$ (SIBFA) (without the $E_{\text {disp }}$ contribution), as they differ by five $\mathrm{kcal} / \mathrm{mole}$ out of 19 . While the ALMOEDA and SIBFA $E_{1}$ values are close for both rings, both $E_{\mathrm{pol}}$ and $E_{\mathrm{ct}}$ (SIBFA) are underestimated by $2-2.5 \mathrm{kcal} / \mathrm{mole}$ each. Thus the much closer agreements between $\Delta E_{\text {tot }}($ SIBFA $)$ and $\Delta E(\omega$ B97X-D) found with these two rings $(<0.3 \mathrm{kcal} / \mathrm{mole}$ out of 39$)$ stem from a compensation of errors, $E_{\text {disp }}$ (SIBFA) having a five $\mathrm{kcal} / \mathrm{mole}$ larger magnitude than the stabilization energy brought upon passing from the HF to the $\omega \mathrm{B} 97 \mathrm{X}-\mathrm{D}$ level. On the other hand, $\triangle E$ (SIBFA) has an only $0.3 \mathrm{kcal} / \mathrm{mole}$ out of 18 smaller magnitude than $\triangle E(\mathrm{HF})$ for the fluoro-chlorobenzene ring of EVG, but this stems from a four $\mathrm{kcal} / \mathrm{mole}$ less repulsive value of $E_{1}(\mathrm{SIBFA})$ than $E_{1}(\mathrm{HF})$, compensating for the four $\mathrm{kcal} / \mathrm{mole}$ undererestimation of $E_{\mathrm{pol}}+E_{\mathrm{ct}}$. Such analyses show the continuous need to check for a term-to-term identification of the SIBFA and QC contributions. Improvements along these lines are being carried out presently, but with correlated multipoles and polarizabilities and on the basis of correlated EDA approaches. Each ligand of interest is probed in a diversity of positions by cationic and dipolar probes (El Khoury et al., 2017; Kwapien et al., 2017) and using an automated procedure which, for each individual contribution, optimizes the relevant parameters by least-squares fit minimizing the error with respect to its QC counterpart (M. Devillers, et al., 2019, unpublished data). 
Table 4 Intermolecular interaction energies $(\mathrm{kcal} / \mathrm{mol})$ of the three halobenzene rings in their own as well as in the other two inhibitor G4/C16 binding sites.

\begin{tabular}{llll} 
& RAL site & EVG site & DTG site \\
\hline$\Delta E$ (B97D3)-RAL & -37.2 & -38.3 & -37.7 \\
$\Delta E$ (B97D3)-EVG & & -39.6 & -40.5 \\
$\Delta E$ (B97D3)-DTG & & -39.4 & -38.0 \\
\hline
\end{tabular}

We have further considered whether the EVG ring preference over the DTG ring could be possibly biased by their initial "anchorings" by their diketoacid connector preventing the displacement of the HC halobenzene connecting bond. For that purpose, we have permuted the DTG and EVG ring positions in the ternary complexes. That is, the HC connecting bond of the DTG ring was superimposed over that of the EVG ring in the EVG complex and its plane then superimposed over the EVG one. We proceeded in a similar fashion to superimpose the EVG ring over the DTG one in the ternary DTG complex. We have also superimposed the RAL ring over the DTG and EVG ones in their ternary complexes. These three "chimeric" complexes were then energy-minimized with the B97-D3 functional, the $\mathrm{HN}_{9}, \mathrm{HN}_{1}$, and $\mathrm{HC}$ connecting bonds remaining unrelaxed. The results are reported in Table 4 . On the one hand, in each binding site, RAL has a consistently lesser binding affinity than EVG and DTG. On the other hand, in the EVG binding site, the DTG-EVG difference is reduced to $0.2 \mathrm{kcal} / \mathrm{mole}$. Conversely, it increased to $2.5 \mathrm{kcal} / \mathrm{mole}$ in the DTG binding site.

This indicates that regardless of the additional interactions involving the diketoacid anchor and DNA, a modulation of the ring affinities can depend on the location of the anchor in the DNA grooves. Thus $\triangle E(\mathrm{QC})$ for EVG binding appears more favored in the DTG site $(-40.5 \mathrm{kcal} / \mathrm{mole})$ than in its own site $(-39.6 \mathrm{kcal} / \mathrm{mole})$. Everything else being equal, this could imply that connecting the diketoacid ring of DTG to the fluoro-chlorobenzene ring of EVG rather than difluorobenzene could improve the drug binding affinity, albeit by a modest amount (one $\mathrm{kcal} / \mathrm{mole}$ out of 40 ). A similar inversion occurs in the case of DTG, with $\Delta E(\mathrm{QC})$ values of -39.4 in the EVG site and -38.0 in its own site, so that the difluorobenzene ring could better contribute to the biding affinity if grafted to the diketoacid of EVG rather than that of DTG.

\section{DISCUSSION}

$\Delta E_{\text {tot }}(\mathrm{SIBFA})$ had values and trends consistent with the $\Delta E(\mathrm{QC})$ ones. However, in the case of the fluoro-chorinated ring of EVG, $\Delta E_{\text {tot }}(\mathrm{SIBFA})$ presently overestimates $\Delta E(\mathrm{QC})$ by amounts in the two out $40 \mathrm{kcal} / \mathrm{mole}$ range. SIBFA is being presently refined using correlated rather than HF-derived multipoles, and its calibration done on the basis of correlated energy decomposition analyses. These should further narrow down the errors with respect to $\Delta E(\mathrm{QC})$ with the appropriate functional, in terms of both the total energy and its individual contributions.

\section{CONCLUSIONS AND PERSPECTIVES}

This study focused on three INSTIs successively used in anti-HIV-1 therapy, RAL, EVG, and DTG. We carried out measurements of their free energies of binding, $\Delta G$, to the 
vDNA end in solution, and unraveled their enthalpy and entropy components in solution. We found that the $\Delta G$ ranking DTG $>$ EVG $>$ RAL parallels that inferred for the intasome (Hightower et al., 2011). The $\Delta G$ ranking is also paralleled by the $\Delta H$ one, a signature for intercalation-driven binding (Chaires, 2006).

We have considered three model ternary complexes, involving G4, C16 and the halobenzene ring of DTG, EVG, and RAL. These were extracted from the corresponding $\mathrm{X}$-ray crystal structures and energy-minimized them by DFT/B97-D3 computations, freezing the $\mathrm{HN}_{9}, \mathrm{HN}_{1}$, and $\mathrm{HC}$ bonds connecting $\mathrm{G} 4, \mathrm{C16}$, and the ring to their DNA and diketoacid anchors. The mono-fluorobenzene ring of RAL had the least favorable $\triangle E(\mathrm{QC})$ values, whatever the DFT functional. However, the chloro-fluorobenzene ring of EVG had more favorable $\Delta E(\mathrm{QC})$ values than the difluorobenzene of DTG, the energy difference being in the range $0.1-1.6 \mathrm{kcal} /$ mole out of 40 . Such a preference could plainly indicate that fluoro-chlorobenzene interacts more favorably with the G4/C16 base pairs than difluorobenzene, on account of its larger sigma-hole as well as more favorable van der Waals dispersion effects. But this leaves open the contribution of the dikeoacid connector in the relative affinities. On the one hand, it contributes interactions of its own with the backbone. But it also anchors the halobenzene ring with respect to the G4/C16 base-pair. Along these lines, it was found that grafting the fluoro-chlorobenzene ring of EVG on the diketoacid arm of DTG resulted, in the DTG binding site, into a one $\mathrm{kcal} / \mathrm{mole}$ $\triangle E(\mathrm{QC})$ gain. A similar gain was observed in the case of the difluorobenzene ring of DTG, if transposed into the EVG binding site.

As put forth in El Hage et al. (2015), it is possible to leverage the "Janus-like" properties of the $\mathrm{CX}$ bond $(\mathrm{X}=\mathrm{F}, \mathrm{Cl}, \mathrm{Br}$ ), electron-deficient along the bond and electron-rich in a cone around it, to target respectively and simultaneously electron-rich and electron-deficient sites of the nucleotide bases. Polarizable molecular mechanics is responsive to the electronic changes brought about by substitutions as these impact the magnitude of both QC-derived distributed multipoles and polarizabilities used to compute the $E_{\mathrm{MTP}}{ }^{*}$ and $E_{\mathrm{pol}}$ contributions. These should enable to fine-tune and further evolve the affinity of halobenzenes for targeted HIV-1 DNA bases.

Along these lines, several novel compounds were recently designed and endowed with significantly more favorable $\Delta E(\mathrm{QC})$ and $\Delta E$ (SIBFA) values than the difluoro benzene ring of DTG and the fluoro-chlorine ring of EVG. They will be reported in a forthcoming paper (P. El Darazi, et al., 2019, unpublished data).

In addition to handling the halobenzene interactions (El Hage et al., 2013, 2014), a further asset of SIBFA and related polarizable potentials (Bell et al., 2016) is the reliable handling of poly-ligated complexes of divalent cations (El Khoury et al., 2017; Kwapien et al., 2017) and interactions involving "discrete" structural waters (De Courcy et al., 2010; Gresh et al., 2011). Such structural motives are also encountered in the intasome-INSTI complexes.

Grounded on these validations, we plan to undertake long-duration polarizable molecular dynamics simulations on a diversity of INSTI complexes with intasome, resorting to the massively parallel computer code Tinker-HP. These will enable to quantify the extent to which the halobenzene-G4/C16 interactions are modulated by the conformational 
flexibilities of each partner within the complex, by the electrostatic potentials and fields exerted by the neighboring INT residues and vDNA bases, and possibly as well by the two neighboring divalent $\mathrm{Mg}(\mathrm{II})$ cations and by the structural waters.

\section{ACKNOWLEDGEMENTS}

We wish to thank the reviewers for constructive comments.

\section{ADDITIONAL INFORMATION AND DECLARATIONS}

\section{Funding}

This work was supported by the Grand Equipement National de Calcul Intensif (GENCI): Institut du Développement et des Ressources en Informatique Scientifique (IDRIS), Centre Informatique de l'Enseignement Supérieur (CINES), France, project No. x2009-075009, and the Centre de Ressources Informatiques de Haute Normandie (CRIHAN, Rouen, France), project 1998053. This work was funded by the Research Council of Saint- Joseph University of Beirut, Lebanon (Project FS71), the Lebanese National Council for Scientific Research, CNRS-L (Project FS80), as well as the French Institute- Lebanon and the French-Lebanese Program CEDRE (Project 35327UJ). This work has received funding from the European Research Council (ERC) under the European Union's Horizon 2020 research and innovation program (grant agreement No 810367), project EMC2.

The funders had no role in study design, data collection and analysis, decision to publish, or preparation of the manuscript.

\section{Grant Disclosures}

The following grant information was disclosed by the authors:

Grand Equipement National de Calcul Intensif (GENCI) Institut du Développement et des Ressources en Informatique Scientifique (IDRIS), Centre Informatique de l'Enseignement Supérieur (CINES), France, project No: x2009-075009.

Centre Régional Infomatique et d'Applications Numériques de Normandie (CRIANN, Rouen, France), project 1998053.

Research Council of Saint- Joseph University of Beirut, Lebanon Project: FS71.

Lebanese National Council for Scientific Research, CNRS-L Project: FS80.

French Institute- Lebanon and the French-Lebanese Program CEDRE Project: 35327UJ. European Research Council (ERC) under the European Union's Horizon 2020 research and innovation program (project EMC2): 810367.

\section{Competing Interests}

The authors declare that they have no competing interests.

\section{Author Contributions}

- Léa El Khoury conceived and designed the experiments, performed the experiments, analyzed the data, contributed reagents/materials/analysis tools, prepared figures and/or tables, performed the computation work, authored or reviewed drafts of the paper, approved the final draft. 
- Krystel El Hage performed the experiments, prepared figures and/or tables, performed the computation work, approved the final draft.

- Jean-Philip Piquemal analyzed the data, performed the computation work, authored or reviewed drafts of the paper, approved the final draft.

- Serge Fermandjian conceived and designed the experiments, analyzed the data, authored or reviewed drafts of the paper, approved the final draft.

- Richard Maroun conceived and designed the experiments, analyzed the data, authored or reviewed drafts of the paper, approved the final draft.

- Nohad Gresh analyzed the data, prepared figures and/or tables, performed the computation work, authored or reviewed drafts of the paper, approved the final draft.

- Zeina Hobaika conceived and designed the experiments, analyzed the data, contributed reagents/materials/analysis tools, prepared figures and/or tables, authored or reviewed drafts of the paper, approved the final draft.

\section{Data Availability}

The following information was supplied regarding data availability:

Raw data is available as Supplemental Files.

\section{Supplemental Information}

Supplemental information for this article can be found online at http://dx.doi.org/10.7717/ peerj-pchem.6\#supplemental-information.

\section{REFERENCES}

Ammar FF, Abdel-Azeim S, Zargarian L, Hobaika Z, Maroun RG, Fermandjian S. 2012. Unprocessed viral DNA could be the primary target of the HIV-1 integrase inhibitor raltegravir. PLOS ONE 7(7):e40223 DOI 10.1371/journal.pone.0040223.

Ammar FF, Hobaika Z, Abdel-Azeim S, Zargarian L, Maroun RG, Fermandjian S. 2016. A targeted DNA substrate mechanism for the inhibition of HIV-1 integrase by inhibitors with antiretroviral activity. FEBS Open Bio 6(4):234-250 DOI 10.1002/2211-5463.12025.

Anstett K, Brenner B, Mesplede T, Wainberg MA. 2017. HIV drug resistance against strand transfer integrase inhibitors. Retrovirology 14(1):36 DOI 10.1186/s12977-017-0360-7.

Balakrishnan M, Yant SR, Tsai L, O’Sullivan C, Bam RA, Tsai A, Niedziela-Majka A, Stray KM, Sakowicz R, Cihlar T. 2013. Non-catalytic site HIV-1 integrase inhibitors disrupt core maturation and induce a reverse transcription block in target cells. PLOS ONE 8(9):e74163 DOI 10.1371/journal.pone.0074163.

Ballantyne AD, Perry CM. 2013. Dolutegravir: first global approval. Drugs 73(14):1627-1637 DOI 10.1007/s40265-013-0121-4.

Bell DR, Qi R, Jing Z, Xiang JY, Mejias C, Schnieders MJ, Ponder JW, Ren P. 2016. Calculating binding free energies of host-guest systems using the AMOEBA polarizable force field. Physical Chemistry Chemical Physics 18(44):30261-30269 DOI 10.1039/C6CP02509A.

Blanco JL, Varghese V, Rhee SY, Gatell JM, Shafer RW. 2011. HIV-1 integrase inhibitor resistance and its clinical implications. Journal of Infectious Diseases 203(9):1204-1214 DOI 10.1093/infdis/jir025. 
Boys SF, Bernardi FD. 1970. The calculation of small molecular interactions by the differences of separate total energies. Some procedures with reduced errors. Molecular Physics 19(4):553-566 DOI 10.1080/00268977000101561.

Bushman FD, Craigie R. 1991. Activities of human immunodeficiency virus (HIV) integration protein in vitro: specific cleavage and integration of HIV DNA. Proceedings of the National Academy of Sciences of the United States of America 88(4):1339-1343 DOI 10.1073/pnas.88.4.1339.

Chaires JB. 2006. A thermodynamic signature for drug-DNA binding mode. Archives of Biochemistry and Biophysics 453(1):26-31 DOI 10.1016/j.abb.2006.03.027.

Chen Q, Buolamwini JK, Smith JC, Li A, Xu Q, Cheng X, Wei D. 2013. Impact of resistance mutations on inhibitor binding to HIV-1 integrase. Journal of Chemical Information and Modeling 53(12):3297-3307 DOI 10.1021/ci400537n.

Clark T, Hennemann M, Murray JS, Politzer P. 2007. Halogen bonding: the $\sigma$-hole. Journal of Molecular Modeling 13(2):291-296 DOI 10.1007/s00894-006-0130-2.

Creuzet S, Langlet J, Gresh N. 1991. Adjustment of the SIBFA method for potential maps to study hydrogen bonding vibrational frequencies. AIP Conference Proceedings 239(1):114.

De Courcy B, Piquemal J-P, Garbay C, Gresh N. 2010. Polarizable water molecules in ligand -macromolecule recognition. Impact on the relative affinities of competing pyrrolopyrimidine inhibitors for FAK kinase. Journal of the American Chemical Society 132(10):3312-3320 DOI 10.1021/ja9059156.

Devereux M, Raghunathan S, Fedorov DG, Meuwly M. 2014. Computationally efficient multipolar model employing distributed charges for molecular dynamics simulations. Journal of Chemical Theory and Computation 10(10):4229-4241 DOI 10.1021/ct500511t.

Dunning TH Jr. 1989. Gaussian basis sets for use in correlated molecular calculations. I. The atoms boron through neon and hydrogen. Journal of Chemical Physics 90(2):1007-1023 DOI 10.1063/1.456153.

El Hage K, Bereau T, Jakobsen S, Meuwly M. 2016. Impact of quadrupolar electrostatics on atoms adjacent to the sigma-hole in condensed-phase simulations. Journal of Chemical Theory and Computation 12(7):3008-3019 DOI 10.1021/acs.jctc.6b00202.

El Hage K, Piquemal JP, Hobaika Z, Maroun RG, Gresh N. 2013. Could an anisotropic molecular mechanics/dynamics potential account for sigma hole effects in the complexes of halogenated compounds? Journal of Computational Chemistry 34(13):1125-1135 DOI 10.1002/jcc.23242.

El Hage K, Piquemal JP, Hobaika Z, Maroun RG, Gresh N. 2014. Substituent-modulated affinities of halobenzene derivatives to the HIV-1 integrase recognition site. Analyses of the interaction energies by parallel quantum chemical and polarizable molecular mechanics. Journal of Physical Chemistry A 118(41):9772-9782 DOI 10.1021/jp5079899.

El Hage K, Piquemal JP, Hobaika Z, Maroun RG, Gresh N. 2015. Could the "Janus-like" properties of the halobenzene $\mathrm{CX}$ bond $(\mathrm{X}=\mathrm{Cl}, \mathrm{Br})$ be leveraged to enhance molecular recognition? Journal of Computational Chemistry 36(4):210-221 DOI 10.1002/jcc.23786.

El Khoury L, Naseem-Khan S, Kwapien K, Hobaika Z, Maroun RG, Piquemal JP, Gresh N. 2017. Importance of explicit smeared lone-pairs in anisotropic polarizable molecular mechanics. Torture track angular tests for exchange-repulsion and charge transfer contributions. Journal of Computational Chemistry 38(22):1897-1920 DOI 10.1002/jcc.24830.

El Khoury L, Piquemal JP, Fermandjian S, Maroun RG, Gresh N, Hobaika Z. 2017. The inhibition process of HIV-1 integrase by diketoacids molecules: understanding the factors governing the better efficiency of dolutegravir. Biochemical and Biophysical Research Communications 488(3):433-438 DOI 10.1016/j.bbrc.2017.05.001. 
Engelman A, Englund G, Orenstein JM, Martin MA, Craigie R. 1995. Multiple effects of mutations in human immunodeficiency virus type 1 integrase on viral replication. Journal of Virology 69(5):2729-2736.

Feller D. 1996. The role of databases in support of computational chemistry calculations. Journal of Computational Chemistry 17(13):1571-1586.

Fontana J, Jurado KA, Cheng N, Ly NL, Fuchs JR, Gorelick RJ, Engelman AN, Steven AC. 2015. Distribution and redistribution of HIV-1 nucleocapsid protein in immature, mature, and integrase-inhibited virions: a role for integrase in maturation. Journal of Virology 89(19):9765-9780 DOI 10.1128/JVI.01522-15.

Frisch MJ, Trucks GW, Schlegel HB, Scuseria GE, Robb MA, Cheeseman JR, Scalmani G, Barone V, Mennucci B, Petersson GA, Nakatsuji H. 2009. Gaussian 09 Revision D. Wallingford: Gaussian Inc.

Garmer DR, Stevens WJ. 1989. Transferability of molecular distributed polarizabilities from a simple localized orbital based method. Journal of Physical Chemistry 93(25):8263-8270 DOI 10.1021/j100362a023.

Goerigk L, Grimme S. 2011. A thorough benchmark of density functional methods for general main group thermochemistry, kinetics, and noncovalent interactions. Physical Chemistry Chemical Physics 13(14):6670-6688 DOI 10.1039/c0cp02984j.

Goethals O, Vos A, Van Ginderen M, Geluykens P, Smits V, Schols D, Hertogs K, Clayton R. 2010. Primary mutations selected in vitro with raltegravir confer large fold changes in susceptibility to first-generation integrase inhibitors, but minor fold changes to inhibitors with second-generation resistance profiles. Virology 402(2):338-346 DOI 10.1016/j.virol.2010.03.034.

Gresh N, Cisneros GA, Darden TA, Piquemal J-P. 2007. Anisotropic, polarizable molecular mechanics studies of inter-, intramolecular interactions, and ligand-macromolecule complexes. A bottom-up strategy. Journal of Chemical Theory and Computation 3(6):1960-1986 DOI 10.1021/ct700134r.

Gresh N, De Courcy B, Piquemal J-P, Foret J, Courtiol-Legourd Ś, Salmon L. 2011. Polarizable water networks in ligand-metalloprotein recognition. Impact on the relative complexation energies of $\mathrm{Zn}$-dependent phosphomannose isomerase with d-Mannose 6-phosphate surrogates. Journal of Physical Chemistry B 115(25):8304-8316 DOI 10.1021/jp2024654.

Grimme S. 2006. Semiempirical GGA-type density functional constructed with a long-range dispersion correction. Journal of Computational Chemistry 27(15):1787-1799 DOI 10.1002/jcc.20495.

Grinsztejn B, Nguyen BY, Katlama C, Gatell JM, Lazzarin A, Vittecoq D, Gonzalez CJ, Chen J, Harvey CM, Isaacs RD. 2007. Safety and efficacy of the HIV-1 integrase inhibitor raltegravir (MK-0518) in treatment-experienced patients with multidrug-resistant virus: a phase II randomised controlled trial. Lancet 369(9569):1261-1269

DOI 10.1016/S0140-6736(07)60597-2.

Hare S, Gupta SS, Valkov E, Engelman A, Cherepanov P. 2010a. Retroviral intasome assembly and inhibition of DNA strand transfer. Nature 464(7286):232-236 DOI 10.1038/nature08784.

Hare S, Smith SJ, Métifiot M, Jaxa-Chamiec A, Pommier Y, Hughes SH, Cherepanov P. 2011. Structural and functional analyses of the second-generation integrase strand transfer inhibitor dolutegravir (S/GSK1349572). Molecular Pharmacology 80(4):565-572

DOI 10.1124/mol.111.073189.

Hare S, Vos AM, Clayton RF, Thuring JW, Cummings MD, Cherepanov P. 2010b. Molecular mechanisms of retroviral integrase inhibition and the evolution of viral resistance. Proceedings of 
the National Academy of Sciences of the United States of America 107(46):20057-20062 DOI 10.1073/pnas.1010246107.

Hazuda DJ, Felock P, Witmer M, Wolfe A, Stillmock K, Grobler JA, Espeseth A, Gabryelski L, Schleif W, Blau C, Miller MD. 2000. Inhibitors of strand transfer that prevent integration and inhibit HIV-1 replication in cells. Science 287(5453):646-650 DOI 10.1126/science.287.5453.646.

Heyduk T, Lee JC. 1990. Application of fluorescence energy transfer and polarization to monitor Escherichia coli cAMP receptor protein and lac promoter interaction. Proceedings of the National Academy of Sciences of the United States of America 87(5):1744-1748 DOI 10.1073/pnas.87.5.1744.

Hightower KE, Wang R, DeAnda F, Johns BA, Weaver K, Shen Y, Tomberlin GH, Carter HL III, Broderick T, Sigethy S, Seki T, Kobayashi M, Underwood MR. 2011. Dolutegravir (S/GSK1349572) exhibits significantly slower dissociation than Raltegravir and Elvitegravir from wild type and integrase inhibitor-resistant HIV-1 integrase-DNA complexes. Antimicrobial Agents and Chemotherapy 55(10):4552-4559 DOI 10.1128/AAC.00157-11.

Hill JJ, Royer CA. 1997. Fluorescence approaches to study of protein-nucleic acid complexation. In: Methods in Enzymology. Vol. 278. Amsterdam: Elsevier, 390-416 DOI 10.1016/S0076-6879(97)78021-2.

Hobaika Z, Zargarian L, Boulard Y, Maroun RG, Mauffret O, Fermandjian S. 2009. Specificity of LTR DNA recognition by a peptide mimicking the HIV-1 integrase $\alpha 4$ helix. Nucleic Acids Research 37(22):7691-7700 DOI 10.1093/nar/gkp824.

Hobaika Z, Zargarian L, Maroun RG, Mauffret O, Burke TR, Fermandjian S. 2010. HIV-1 integrase and virus and cell DNAs: complex formation and perturbation by inhibitors of integration. Neurochemical Research 35(6):888-893 DOI 10.1007/s11064-009-0098-2.

Horn PR, Mao Y, Head-Gordon M. 2016. Probing non-covalent interactions with a second generation energy decomposition analysis using absolutely localized molecular orbitals. Physical Chemistry Chemical Physics 18(33):23067-23079 DOI 10.1039/C6CP03784D.

Ibrahim MA. 2011. Molecular mechanical study of halogen bonding in drug discovery. Journal of Computational Chemistry 32(12):2564-2574 DOI 10.1002/jcc.21836.

Ikeda T, Nishitsuji H, Zhou X, Nara N, Ohashi T, Kannagi M, Masuda T. 2004. Evaluation of the functional involvement of human immunodeficiency virus type 1 integrase in nuclear import of viral cDNA during acute infection. Journal of Virology 78(21):11563-11573 DOI 10.1128/JVI.78.21.11563-11573.2004.

Jorgensen WL, Schyman P. 2012. Treatment of halogen bonding in the OPLS-AA force field: application to potent anti-HIV agents. Journal of Chemical Theory and Computation 8(10):3895-3901 DOI 10.1021/ct300180w.

Khaliullin RZ, Cobar EA, Lochan RC, Bell AT, Head-Gordon M. 2007. Unravelling the origin of intermolecular interactions using absolutely localized molecular orbitals. Journal of Physical Chemistry A 111(36):8753-8765 DOI 10.1021/jp073685z.

Koelsch KK, Cooper DA. 2009. Integrase inhibitors in salvage therapy regimens for HIV-1 infection. Current Opinion in HIV and AIDS 4(6):518-523

DOI 10.1097/COH.0b013e328331b526.

Kolar M, Hobza P. 2012. On extension of the current biomolecular empirical force field for the description of halogen bonds. Journal of Chemical Theory and Computation 8:1325-1333 DOI 10.1021/ct2008389.

Kwapien K, Damergi D, Nader S, El Khoury L, Hobaika Z, Maroun RG, Piquemal J-P, Gavara L, Berthomieu D, Hernandez J-F, Gresh N. 2017. Calibration of 1,2,4 triazole-3-thione, 
an original $\mathrm{Zn}$-binding group of metallo- $\beta$-lactamase inhibitors. Validation of a polarizable MM/MD potential by quantum chemistry. Journal of Physical Chemistry B 121(26):6295-6312 DOI 10.1021/acs.jpcb.7b01053.

LaFemina RL, Callahan PL, Cordingley MG. 1991. Substrate specificity of recombinant human immunodeficiency virus integrase protein. Virology 65(10):5624-5630.

Lagardère L, Jolly L-H, Lipparini F, Aviat F, Stamm B, Jing ZF, Harger M, Torabifard H, Cisneros GA, Schnieders MJ, Gresh N, Maday Y, Ren PY, Ponder JW, Piquemal J-P. 2018. Tinker-HP: a massively parallel molecular dynamics package for multiscale simulations of large complex systems with advanced point dipole polarizable force fields. Chemical Science 9(4):956-972 DOI 10.1039/C7SC04531J.

Lesbats P, Engelman AN, Cherepanov P. 2016. Retroviral DNA integration. Chemical Reviews 116(20):12730-12757 DOI 10.1021/acs.chemrev.6b00125.

Li X, Krishnan L, Cherepanov P, Engelman A. 2011. Structural biology of retroviral DNA integration. Virology 411(2):194-205 DOI 10.1016/j.virol.2010.12.008.

Mardirossian N, Head-Gordon M. 2014. $\omega \mathrm{B} 97 \mathrm{X}-\mathrm{V}$ : a 10-parameter, range-separated hybrid, generalized gradient approximation density functional with nonlocal correlation, designed by a survival-of-the-fittest strategy. Physical Chemistry Chemical Physics 16(21):9904-9924 DOI 10.1039/c3cp54374a.

Marinello J, Marchand C, Mott BT, Bain A, Thomas CJ, Pommier Y. 2008. Comparison of raltegravir and elvitegravir on HIV-1 integrase catalytic reactions and on a series of drug-resistant integrase mutants. Biochemistry 47(36):9345-9354 DOI 10.1021/bi800791q.

Métifiot M, Marchand C, Maddali K, Pommier Y. 2010. Resistance to integrase inhibitors. Viruses 2(7):1347-1366 DOI 10.3390/v2071347.

Métifiot M, Marchand C, Pommier Y. 2013. HIV integrase inhibitors: 20-year landmark and challenges. Advances in Pharmacology 67:75-105 DOI 10.1016/B978-0-12-405880-4.

Naeger LK, Harrington P, Komatsu T, Deming D. 2016. Effect of dolutegravir functional monotherapy on HIV-1 virological response in integrase strand transfer inhibitor resistant patients. Antiviral Therapy 21(6):481-488 DOI 10.3851/IMP3033.

Osterholzer DA, Goldman M. 2014. Dolutegravir: a next-generation integrase inhibitor for treatment of HIV infection. Clinical Infectious Diseases 59(2):265-271 DOI $10.1093 / \mathrm{cid} / \mathrm{ciu} 221$.

Piquemal JP, Gresh N, Giessner-Prettre C. 2003. Improved formulas for the calculation of the electrostatic contribution to the intermolecular interaction energy from multipolar expansion of the electronic distribution. Journal of Physical Chemistry A 107(48):10353-10359 DOI 10.1021/jp035748t.

Politzer P, Murray JS, Clark T. 2010. Halogen bonding: an electrostatically-driven highly directional noncovalent interaction. Physical Chemistry Chemical Physics 12(28):7748-7757 DOI 10.1039/c004189k.

Pommier Y, Johnson AA, Marchand C. 2005. Integrase inhibitors to treat HIV/AIDS. Nature Reviews Drug Discovery 4(3):236-248 DOI 10.1038/nrd1660.

Rhee SY, Taylor J, Fessel WJ, Kaufman D, Towner W, Troia P, Ruane P, Hellinger J, Shirvani V, Zolopa A, Shafer RW. 2010. HIV-1 protease mutations and protease inhibitor cross-resistance. Antimicrobial Agents and Chemotherapy 54(10):4253-4261 DOI 10.1128/AAC.00574-10.

Schmidt MW, Baldridge KK, Boatz JA, Elbert ST, Gordon MS, Jensen JH, Koseki S, Matsunaga N, Nguyen KA, Su S, Windus TL, Dupuis M, Montgomery JA. 1993. 
General atomic and molecular electronic structure system. Journal of Computational Chemistry 14(11):1347-1363 DOI 10.1002/jcc.540141112.

Shao Y, Gan Z, Epifanovsky E, Gilbert TBA, Wormit M, Kussmann J, Lange AW, Behn A, Deng J, Feng X, Ghosh D, Goldey M, Horn PR, Jacobson LD, Kaliman I, Kus T, Landau A, Liu J, Proynov EI, Richard RM, Steele RP, Sundstrom EJ, Woodcock HL III, Zimmerman PM, Zuev D, Albrecht B, Alguire E, Baeppler SA, Barton D, Bernard YA, Berquist EJ, Bravaya KB, Burton H, Casanova D, Chang C-M, Chen Y, Chien A, Closser KD, Coons MP, Dasgupta S, Dempwolff AL, Diedenhofen M, Do H, Edgar RG, Fang P-T, Fatehi S, Feng Q, Fosso-Tande J, Ge Q, Ghysels A, Gidofalvi G, Gomes J, Gonthier J, Gunina A, Hanson-Heine MWD, Harbach PHP, Hauser AW, Herr JE, Hohenstein EG, Holden ZC, Hui K, Jagau T-C, Ji H, Kaduk B, Khistyaev K, Kim J, Klunzinger P, Koh K, Kosenkov D, Koulias L, Kowalczyk T, Krauter CM, Kunitsa A, Lao KU, Laurent A, Lawler KV, Lee J, Lefrancois D, Lehtola S, Levine DS, Li Y-P, Lin Y-S, Liu F, Livshits E, Luenser A, Manohar P, Mansoor E, Manzer SF, Mao S-P, Mao Y, Mardirossian N, Marenich AV, Markovich T, Martinez-Martinez LA, Maurer sA, Mayhall NJ, McKenzie SC, Mewes J-M, Morrison AF, Mullinax JW, Nanda K, Nguyen-Beck TS, Olivares-Amaya R, Parkhill A, Perrine TM, Plasser F, Prager S, Prociuk A, Ramos E, Rehn DR, Rob F, Schneider M, Sergueev N, Sharada SM, Sharma S, Small DW, Stauch T, Stein T, Chuan Su Y-C, Thom AJW, Tkatchenko A, Tsuchimochi T, Vogt L, Vydrov O, Watson MA, Wenzel J, de Wergifosse M, Wesolowski TA, White A, Witte J, Yamada A, Yang J, Yao K, Yeganeh S, Yost SR, You Z-Q, Zech A, Ying Zhang I, Zhang X, Zhao Y, Zhu Y, Brooks BR, Chan GKL, Cramer CJ, Gordon MS, Hehre WJ, Klamt A, Schmidt MW, Sherrill CD, Truhlar DG, Aspuru-Guzik A, Baer R, Bell AT, Besley NA, Chai J-D, DiStasio RA Jr, Dreuw A, Dunietz BD, Furlani TR, Hsu C-P, Jung Y, Kong J, Lambrecht DS, Liang W-Z, Ochsenfeld C, Rassolov A, Slipchenko LV, Subotnik JE, Van Voorhis T, Herbert JM, Krylov AI, Gill PMW, Head-Gordon M. 2015. Advances in molecular quantum chemistry contained in the Q-Chem 4 program package. Molecular Physics 113(2):184-215.

Sherman PA, Dickson ML, Fyfe JA. 1992. Human immunodeficiency virus type 1 integration protein: DNA sequence requirements for cleaving and joining reactions. Virology 66(6):3593-3601.

Simon S, Duran M, Dannenberg JJ. 1996. How does basis set superposition error change the potential surfaces for hydrogen-bonded dimers? Journal of Chemical Physics 105(24):11024-11031 DOI 10.1063/1.472902.

Stone AJ. 1981. Distributed multipole analysis, or how to describe a molecular charge distribution. Chemical Physics Letters 83(2):233-239 DOI 10.1016/0009-2614(81)85452-8.

Stone AJ, Alderton M. 1985. Distributed multipole analysis: methods and applications. Molecular Physics 56(5):1047-1064 DOI 10.1080/00268978500102891.

Tantillo C, Ding J, Jacobo-Molina A, Nanni RG, Boyer PL, Hughes SH, Pauwels R, Andries K, Janssen PA, Arnold E. 1994. Locations of anti-AIDS drug binding sites and resistance mutations in the three-dimensional structure of HIV-1 reverse transcriptase: implications for mechanisms of drug inhibition and resistance. Journal of Molecular Biology 243(3):369-387 DOI 10.1006/jmbi.1994.1665.

Vandekerckhove L. 2010. GSK-1349572, a novel integrase inhibitor for the treatment of HIV infection. Current Opinion in Investigational Drugs 11(2):203-212.

Vigné-Maeder F, Claverie P. 1988. The exact multicenter multipolar part of a molecular charge distribution and its simplified representations. Journal of Chemical Physics 88(8):4934-4948

DOI 10.1063/1.454705. 
Wainberg MA, Han YS. 2015. Will drug resistance against dolutegravir in initial therapy ever occur? Frontiers in Pharmacology 6:90 DOI 10.3389/fphar.2015.00090.

Wittkop L, Breilh D, Da Silva D, Duffau P, Mercie P, Raymond I, Anies G, Fleury H, Saux M-C, Dabis F, Fagard C, Thiebaut R, Masquelier B, Pellegrin I. 2009. Virological and immunological response in HIV-1-infected patients with multiple treatment failures receiving raltegravir and optimized background therapy, ANRS CO3 Aquitaine Cohort. Journal of Antimicrobial Chemotherapy 63(6):1251-1255 DOI 10.1093/jac/dkp114.

Zargarian L, Benleumi MS, Renisio JG, Merad H, Maroun RG, Wieber F, Mauffret O, Porumb H, Troalen F, Fermandjian S. 2003. Strategy to discriminate between high and low affinity bindings of human immunodeficiency virus, type 1 integrase to viral DNA. Journal of Biological Chemistry 278(22):19966-19973 DOI 10.1074/jbc.M211711200.

Zhao XZ, Smith SJ, Maskell DP, Métifiot M, Pye VE, Fesen K, Marchand C, Pommier Y, Cherepanov P, Hughes SH, Burke TR Jr. 2017. Structure-guided optimization of HIV integrase strand transfer inhibitors. Journal of Medicinal Chemistry 60(17):7315-7332 DOI 10.1021/acs.jmedchem.7b00596. 